\title{
Treinamento de dupla tarefa associado a estratégias cognitivas de associação e imagem mental: impacto no equilíbrio, cognição e na saúde mental de idosos
}

\author{
Dual-task training associated with cognitive association and mental image strategies: impact on
}

\author{
balance, cognition and mental health in the elderly
}

Entrenamiento de doble tarea asociado a la asociación cognitiva y las estrategias de imagen mental: impacto en el equilíbrio, cognición y la salud mental en los ancianos

\author{
Caroline Lacerda Hayashi \\ ORCID: https://orcid.org/0000-0002-8253-0755 \\ Centro Universitário de Pato de Minas, Brasil \\ E-mail: carolzina_lacerda@hotmail.com \\ Thiago Henrique Ferreira Vasconcellos \\ ORCID: https://orcid.org/0000-0002-7613-6022 \\ Centro Universitário de Pato de Minas, Brasil \\ E-mail: thiagov@unipam.edu.br \\ Daniel Vicentini de Oliveira \\ ORCID: https://orcid.org/0000-0002-0272-9773 \\ Universidade Cesumar, Brasil \\ E-mail: d.vicentini@hotmail.com \\ José Roberto Andrade do Nascimento Júnior \\ ORCID: https://orcid.org/0000-0003-3836-6967 \\ Universidade Federal do Vale do São Francisco, Brasil \\ E-mail: jroberto.jrs01@gmail.com \\ Maura Fernandes Franco \\ ORCID: https://orcid.org/0000-0002-6588-7920 \\ Universidade Estadual de Campinas, Brasil \\ E-mail: mauraffranco@gmail.com \\ Jéssica Karen Alves Nogueira \\ ORCID: https://orcid.org/0000-0002-5835-3621 \\ Centro Universitário de Pato de Minas, Brasil \\ E-mail: jessicakn@unipam.edu.br
}

\begin{abstract}
Resumo
Este estudo teve como objetivo avaliar a eficácia de um protocolo de dupla tarefa (DT), associado a estratégias cognitivas de associação e imagem mental no equilíbrio, cognição, ansiedade e nos sintomas depressivos de idosos. Trata-se de um estudo quase-experimental, o qual 10 idosos participaram de um programa de atividades de DT por seis semanas. Foram utilizados os testes de equilíbrio do Short Physical Performance Battery (SPBB), o MINI-ACE, MACQ, Escala de Depressão Geriátrica e o Inventário de Ansiedade Geriátrica. A análise dos dados foi conduzida por meio dos testes de Shapiro-Wilk, $\mathrm{t}$ de student dependente e o $d$ de Cohen $(\mathrm{p}<0,05)$. Não houve diferença significativa ( $\mathrm{p}>$ $0,05)$ nas habilidades cognitivas, nos sintomas depressivos e nos sintomas de ansiedade entre o primeiro e último encontro de intervenção em DT. Verificou-se que apenas o desempenho em equilíbrio apresentou alteração entre o primeiro $(\mathrm{x}=10,50)$ e último $(\mathrm{x}=11,10)$ encontro de intervenção, manifestando significância estatisticamente significativa $(\mathrm{p}=0,005)$, e magnitude da diferença média $(\mathrm{d}=0,69)$. Concluiu-se que o programa de intervenção em DT por seis semanas provocou efeito positivo somente sobre o desempenho de equilíbrio dos idosos.
\end{abstract}

Palavras-chave: Atividade motora; Cognição; Depressão; Exercício.

\begin{abstract}
This study aimed to evaluate the effectiveness of a dual-task (DT) protocol, associated with cognitive association strategies and mental image on balance, cognition, anxiety and depressive symptoms in the elderly. This is a quasiexperimental study, in which 10 elderly people participated in a DT activity program for six weeks. Short Physical Performance Battery (SPBB), MINI-ACE, MAC-Q, Geriatric Depression Scale and Geriatric Anxiety Inventory tests were used. Data analysis was conducted using the Shapiro-Wilk, dependent student $t$ and Cohen $d$ tests $(p<0.05)$. There was no significant difference $(p>0.05)$ in cognitive skills, depressive symptoms and anxiety symptoms between the first and last DT intervention encounter. It was found that only performance in balance showed changes between the first $(x=10.50)$ and last $(x=11.10)$ intervention encounter, showing statistically significant significance $(\mathrm{p}=0.005)$,
\end{abstract}


and magnitude of the mean difference $(\mathrm{d}=0.69)$. It was concluded that the intervention program in DT for six weeks had a positive effect only on the balance performance of the elderly.

Keywords: Motor activity; Cognition; Depression; Exercise.

\section{Resumen}

Este estudio tuvo como objetivo evaluar la efectividad de un protocolo de doble tarea (DT), asociado a estrategias de asociación cognitiva e imagen mental sobre el equilibrio, la cognición, la ansiedad y los síntomas depresivos en el anciano. Se trata de un estudio cuasi-experimental, en el que 10 personas mayores participaron en un programa de actividades de DT durante seis semanas. Se utilizaron pruebas de Short Physical Performance Battery (SPBB), MINIACE, MAC-Q, Escala de Depresión Geriátrica e Inventario de Ansiedad Geriátrica. El análisis de los datos se realizó mediante las pruebas de Shapiro-Wilk, $t$ de Student dependiente y d de Cohen $(p<0,05)$. No hubo diferencia significativa ( $p>0,05)$ en las habilidades cognitivas, los síntomas depresivos y los síntomas de ansiedad entre el primer y el último encuentro de intervención de DT. Se encontró que solo el desempeño en equilibrio mostró cambios entre el primer $(\mathrm{x}=$ 10,50) y el último $(\mathrm{x}=11,10)$ encuentro de intervención, mostrando significancia estadísticamente significativa $(\mathrm{p}=$ $0,005)$ y magnitud de la diferencia media $(\mathrm{d}=0,69)$. Se concluyó que el programa de intervención en DT durante seis semanas tuvo un efecto positivo solo en el desempeño del equilibrio de los ancianos.

Palabras clave: Actividad motora; Cognición; Depresión; Ejercicio.

\section{Introdução}

Como consequência do processo de envelhecimento, os indivíduos apresentam inúmeras alterações biológicas, psicológicas, sociais e cognitivas que, em conjunto ou não, geram déficits funcionais (Fechine \& Trompieri, 2015). Dentre as alterações biológicas/físicas, há aquelas relacionadas aos sistemas visual, auditivo, esquelético, articular, muscular e vestibular que podem impactar na diminuição do equilíbrio dos idosos (Maceno, Hermano \& Costa, 2018).

Em relação as alterações cognitivas, destaca-se que podem haver diminuição normal na atenção, concentração, memória e fluência verbal, dentre outras habilidades, mas que não chegam a modificar a funcionalidade e qualidade de vida do idoso, porém, devem ser consideradas. Quando essas alterações excedem o normal, aumenta-se o risco de demências (Kim, Kim, Chang, Lee \& Bae, 2020).

Importante destacar que os déficits cognitivos são uma parte potencial do quadro clínico da depressão, especialmente quando se trata de depressão tardia (Klojcnik, Kavcic \&Vukman, 2017). E o próprio processo de envelhecimento pode aumentar o risco de algumas pessoas desenvolverem sintomas depressivos, devido as diversas alterações e acontecimentos desta etapa da vida (Casey, 2017).

Outra alteração de humor, além dos sintomas depressivos, que pode surgir em idosos é a ansiedade. Clinicamente, os sintomas de ansiedade podem ser mais difíceis de detectar em adultos mais velhos que são menos precisos na identificação dos sintomas de ansiedade e tendem a minimizar os sintomas e atribuí-los à doença física. Já se sabe que há relação entre a ansiedade e seus transtornos na vida adulta ao aumento da morbidade e mortalidade, especialmente relacionadas a uma carga cardiovascular mais elevada e um declínio cognitivo aumentado (Andreescu \& Lee, 2020). Portanto, também é uma variável psicológica a ser investigada na população idosa.

Com base nestas informações, o exercício físico exerce um papel fundamental na prevenção, na promoção de saúde e no tratamento das disfunções apresentadas pelos idosos (Araujo, Fló, Michels, 2010). E dentre os recursos propostos pelos profissionais do exercício, a dupla tarefa (DT) é capaz de minimizar ou ainda evitar altos níveis de incapacidade funcional e cognitiva em idosos (Varela-Vásquez, Minobes-Molina \& Jerez-Roig, 2020).

A DT é descrita na literatura como a realização de uma tarefa inicial (motora ou cognitiva), no qual o indivíduo devese concentrar e destinar o seu foco e, em seguida, é associada uma segunda atividade (também motora ou cognitiva) que deve ser executada ao mesmo tempo (Fatori, Leite, Souza \& Patrizzi, 2015). E, em alguns casos, a realização de DT pode ser prejudicada quando as ações exigidas excedem a capacidade do sistema cognitivo (Bowen. Wenman Mickelborough, Foster, Hill \& Tallis, 2001) ou ainda quando resultam no envolvimento simultâneo de circuitos neurais específicos às duas ações (Fatori, 
Leite, Souza \& Patrizzi, 2015).

O uso do paradigma de treinamento de DT para aumentar a estabilidade postural em pacientes com problemas de equilíbrio, por exemplo, é uma área de interesse emergente. Os efeitos diferenciais das tarefas duplas e do treinamento DT na estabilidade postural ainda permanecem obscuros (Ghai, Ghai \& Effenberg, 2017). Nos protocolos de intervenção em DT, a tarefa cognitiva adicionada a motora gera déficits na estabilidade postural do indivíduo, propiciando assim o treinamento do equilíbrio no idoso (Wiel, Gussekloo, Craen, Exel, Bloem, Westendorp, 2003).

As intervenções em DT parecem melhorar também questões cognitivas gerais (Oliva, Machado, Rodrigues, Leão \& Monteiro Júnior, 2021), assim como como a memória de trabalho (Norouzi, Vaezmosavi, Gerber, Puhse \& Brand, 2019), mas ainda não se tem muitas evidências do impacto da DT nos sintomas depressivos e na ansiedade de idosos.

Outra prática interventiva para idosos e que se destaca são as psicoeducativas, como as estratégias cognitivas de associação e imagem mental. Nossa hipótese é de que um programa psicoeducacional voltado para a melhora cognitiva, associado ao exercício de DT, pode promover uma melhora no equilíbrio, cognição, sintomas depressivos e ansiedade em idosos. Dada a eficácia a curto prazo e a viabilidade desse tipo de intervenção, os profissionais em diferentes ambientes de serviço clínico e social devem considerar a implementação dos programas como parte da orientação de serviço da equipe e dos usuários (Mak, Chan \& Yau, 2019).

Diante disso, considerando o feito diferencial das PP, associadas as atividades de DT, fundamental para ser compreendido no planejamento de pesquisas ou intervenções futuras para melhorar o desempenho de DT da maioria das atividades da vida diária pretendeu-se com esse estudo avaliar a eficácia de um protocolo DT, associado a estratégias cognitivas de associação e imagem mental no equilíbrio, cognição, ansiedade e nos sintomas depressivos de idosos.

\section{Metodologia}

Trata-se de um estudo quase-experimental, aprovado pelo Comitê de Ética em Pesquisa do UNIPAM, sob protocolo no 3.349.5513.

\subsection{Participantes}

A amostra, escolhida de forma intencional e por conveniência, foi constituída de 10 idosos pertencentes à disciplina Fisioterapia e Qualidade de Vida do programa de extensão "UNIPAM Sênior" do Centro Universitário de Patos de Minas UNIPAM.

Os critérios de inclusão para pesquisa foram: ter idade igual ou superior a 60 anos; estar matriculado na disciplina. Já os critérios de exclusão foram idosos que apresentassem possível déficit cognitivo, avaliado por meio do Mini Exame do Estado Mental (MEEM) (Folstein, Folstein \& McHugh, 1975; Brucki, Nitrini, Caramelli, Bertolucci \& Okamoto 2003).

Foram excluídos também os idosos em uso de cadeira de rodas; sequelas graves de acidente vascular encefálico com perda localizada de força e/ou afasia; doença de Parkinson em estágio grave ou instável com comprometimentos graves da motricidade, da fala ou da afetividade; idosos com graves déficits de audição ou de visão que dificultassem fortemente a comunicação; idosos que apresentassem alterações como dor significante, dor crônica ortopédica, ou deformidades articulares; e idosos que se ausentassem de três sessões consecutivas ou alternadas de intervenção.

\subsection{Instrumentos}

Dentro dos aspectos motores, a variável equilíbrio foi avaliada por meio do instrumento Short Physical Performance Battery (SPBB) (Nakano, 2007), que compreende um conjunto de testes em que o idoso deveria: ficar em pé com os pés juntos; 
em pé com um pé parcialmente à frente, por 10 segundos; e em pé com um pé à frente, por 10 segundos. O escore é zero quando ele se mostra incapaz de manter-se em equilíbrio na primeira posição pelo tempo estipulado. Caso consiga permanecer na primeira posição, mas seja incapaz de manter a segunda, o escore é um ponto. Atribui-se escore dois àquele que conseguisse permanecer na segunda posição, mas não suporta mais de três segundos na terceira. Atribuiu-se escore três quando ele permanecesse na terceira posição por um tempo de três a nove segundos. Atribuiu-se o escore máximo de quatro pontos caso ele conseguisse ficar na terceira posição, por 10 segundos (Nakano, 2007).

O MINI-ACE foi utilizado para mensurar atenção, memória imediata, fluência verbal, habilidade visuoespacial, memória tardia. Consiste em um teste de avaliação cognitiva breve composto de cinco itens, com pontuação máxima de 30 pontos e um tempo de administração de cinco minutos (Miranda, Brucki \& Yassuda, 2018).

Também foi utilizado o MAC-Q, composto por seis questões relacionadas ao funcionamento da memória em atividades cotidianas, como: habilidade para guardar números de telefone de pessoas conhecidas, capacidade para guardar nomes de pessoas que acabou de conhecer, dentre outras. As respostas são pontuadas em uma escala de cinco pontos, os quais variam de "muito melhor agora" até "muito pior agora". O escore total varia entre 7 a 30 pontos, sendo que quanto maior o escore, maior a intensidade de queixa em relação à memória (Crook, Feher \& Larrabe, 1992).

Para verificação dos indicativos de depressão foi aplicado a Escala de Depressão Geriátrica (GDS) que foi criada por Yesavage, Brink, Rose, Lum, Huang, Adey e Leirer (1982) e validada para o Brasil por Paradela, Lourenço \& Veras (2005). A GDS, versão curta, é um teste para detecção de sintomas depressivos no idoso, com 15 perguntas negativas/afirmativas onde o resultado de cinco ou mais pontos aponta indicativos de depressão

O instrumento utilizado para mensurar os sintomas da ansiedade no grupo de idosas foi o Inventário de Ansiedade Geriátrica (GAI), desenvolvido por Pachana Byrne, Siddle, Koloski, Harley e Arnold (2007) e adaptado ao contexto brasileiro por Martiny, Silva, Nardi e Pachana (2011). O inventário é composto por 20 itens dicotômicos no qual o respondente deve escolher "concordo" ou "discordo" como resposta para as afirmações apresentadas. Quanto maior o escore, maior os sintomas de ansiedade. Escores acima de 10 pontos, indicam suspeita de ansiedade.

\subsection{Intervenção}

Os idosos participaram de quatro sessões de intervenção, sendo uma sessão por semana, totalizando quatro semanas. Durante o período de intervenção os participantes foram orientados a manter suas atividades habituais e não iniciarem outros programas de exercício físico.

Cada sessão foi constituída apresentação sobre as estratégias cognitivas, por meio de PP (1 hora de duração), seguida de exercícios de DT intercalados ao treinamento funcional de fisioterapia. Cada sessão teve uma hora de duração.

As PP foram realizadas por estagiários de psicologia, compostas por um vitalizador ou quebra gelo com intuito de "aquecer" o comportamento dos participantes para a próxima etapa. A tarefa central (ou dinâmica central) foi composta por uma atividade lúdica, utilizando recursos manuais, constituídos de exercícios de categorização (organização de informações por similaridade) e associação (gravação de informações com base em conhecimentos prévios). Posteriormente, um espaço para a discussão das atividades realizadas foi propiciado, com o intuito de favorecer reflexão sobre os exercícios, em associação com as atividades do dia-a-dia dos participantes.

Os exercícios que constituíram a DT foram compostos de atividades para membro superior (MMSS) e membro inferior (MMII) com base em uma pista com divisões da área total em "blocos" simulando um circuito. A pista de MMSS e MMII foi marcada com uma fita isolante em uma sala de dança $(20 \times 40 \mathrm{~m})$. Os participantes receberam um treinamento sobre como participar do circuito na primeira sessão de atendimento. Sempre que houvesse um erro ou o participante ficasse parado por não saber o que fazer, o erro será anotado e os profissionais e/ou estagiários de fisioterapia explicavam qual forma correta para 
executar a ação.

Intercalado às pistas de MMII e MMSS havia estímulos visuais coloridos (cones - pista de MMII, fita adesiva - pista de MMSS), em que cada cor representava uma série de exercícios diferentes. Os estímulos estavam acompanhados de uma placa/cartão com o desenho do exercício proposto, seguido de comando verbal dos profissionais e/ou estagiários de fisioterapia sobre as atividades cognitivas a serem realizadas (categorização ou associação).

\subsection{Análise estatística}

A análise dos dados foi realizada por meio do Software SPSS 23.0, mediante estatística descritiva e inferencial. Foi utilizado frequência e percentual como medidas descritivas para as variáveis categóricas. Para as variáveis numéricas, inicialmente foi verificada a normalidade dos dados por meio do teste de Shapiro-wilk. Como os dados apresentaram distribuição normal, foram utilizadas a Média (x) e o Desvio-padrão (dp) como medidas de tendência central e dispersão. Para verificar a efetividade da DT nos aspectos cognitivos (atenção, memória imediata, fluência verbal, habilidade visuoespacial e memória tardia), psicológicos (sintomas depressivos e de ansiedade) e motores (equilíbrio), foi analisada a diferença de média pós e préintervenção, por meio teste de $\mathrm{t}$ de student dependente. Foi calculado o "d de Cohen" para a estimativa de tamanho do efeito, classificando os resultados em pequeno $(0,20$ - 0,49), médio $(0,50-0,79)$ e grande $(>0,80)($ Cohen, 1988; Rosenthal, 1996). A significância adotada foi de $\mathrm{p}<0,05$.

\section{Resultados}

Conforme a Tabela 1 , dos 10 participantes da pesquisa, houve predomínio do sexo feminino (80\%). Os idosos apresentaram média de idade de 67,72 anos (dp =7,67) média de 11,20 anos de escolaridade (dp =6,75), dos quais, 40\% afirmam possuir ensino fundamental incompleto, bem como, $40 \%$ ensino superior incompleto a completo. Em se tratando do status conjugal observou-se um maior percentual de idosos casados $(50,00 \%)$ e viúvos $(30,00 \%)$. Adicionalmente, o arranjo domiciliar era composto pela presença do marido/mulher, companheiro/a $(50,00 \%)$ ou sozinhos/as $(30,00 \%)$.

Tabela 1: Indicadores sociodemográficos e de saúde da amostra.

\begin{tabular}{lll}
\hline VARIÁVEIS & $\boldsymbol{f}$ & $\boldsymbol{\%}$ \\
\hline Sexo & & 20,0 \\
$\quad$ Masculino & 2 & 80,0 \\
$\quad$ Feminino & 8 & 50,0 \\
Estado civil & 5 & 10,0 \\
$\quad$ Casados & 1 & 10,0 \\
$\quad$ Solteiros & 1 & 30,0 \\
$\quad$ Divorciado/Separado & 3 & 30,0 \\
$\quad$ Viúvo & & 50,0 \\
Arranjo familiar & 3 & 20,0 \\
$\quad$ Sozinhos & 5 & \\
$\quad$ Com cônjuge & 2 & 40,0 \\
$\quad$ Filhos ou enteados & & 20,0 \\
Escolaridade & 4 & 10,0 \\
$\quad$ Ensino Fundamental Incompleto & 2 & 30,0 \\
$\quad$ Ensino Médio Completo & 1 & 10,0 \\
$\quad$ Ensino Superior Incompleto & 3 & 20,0 \\
$\quad$ Ensino Superior Completo & & 70,0 \\
Tipo de prática de atividade física & 1 & 2 \\
$\quad$ Vigorosa & 7 & \\
$\quad$ Moderada & 2 & \\
$\quad$ Leve &
\end{tabular}

Fonte: Autores. 
Os dados da Tabela 2 evidenciam que não houve diferença significativa nas habilidades cognitivas entre o primeiro e último encontro de intervenção em DT: atenção $(p=0,509)$; memória imediata $(p=0,343)$; fluência verbal $(p=0,443)$; habilidade visuoespacial ( $\mathrm{p}=0,555)$; memória tardia $(\mathrm{p}=0,11)$; cognição global $(\mathrm{p}=0,217)$; e auto percepção sobre o desempenho de memória $(\mathrm{p}=0,854$ e $\mathrm{p}=0,092)$.

Tabela 2: Efetividade da DT nas habilidades cognitivas, cognição global e autopercepção de memória.

\begin{tabular}{lccccc}
\hline \multirow{2}{*}{ Variáveis } & Pré-teste & Pós-teste & & p-valor & d \\
\cline { 2 - 3 } & $\mathbf{x}(\mathbf{d p})$ & $\mathbf{x}(\mathbf{d p})$ & & & \\
\hline Atenção & $3,70(0,48)$ & $3,50(0,71)$ & & 0,509 & 0,33 \\
Memória imediata & $6,10(0,99)$ & $6,40(0,70)$ & & 0,343 & 0,35 \\
Linguagem & $4,40(1,51)$ & $4,60(1,43)$ & & 0,443 & $0 ., 13$ \\
Habilidade visuoespacial & $4,40(1,08)$ & $4,20(1,40)$ & & 0,555 & 0,15 \\
Memória tardia & $3,90(2,69)$ & $5,00(2,31)$ & & 0,111 & 0,44 \\
Cognição global & $22,50(4,93)$ & $23,70(4,90)$ & & 0,217 & 0,24 \\
MAC-Q & $25,50(5,89)$ & $25,20(4,26)$ & & 0,854 & 0,06 \\
MACNAIR & $10,40(7,63)$ & $6,70(5,58)$ & & 0,092 & 0,55 \\
\hline
\end{tabular}

*Diferença significativa $(\mathrm{p}<0,05)$ - Teste $\mathrm{t}$ de student dependente. Fonte: Autores.

Conforme os resultados da Tabela 3, nota-se que não foi encontrada diferença significativa entre o primeiro e último encontro de intervenção em DT nos sintomas depressivos $(p=0,370)$ e sintomas de ansiedade $(p=1,00)$.

Tabela 3: Efetividade da DT em humor.

\begin{tabular}{lccccc}
\hline \multirow{2}{*}{ Variáveis } & Pré-teste & Pós-teste & & p-valor & d \\
\cline { 2 - 3 } & $\mathbf{x}(\mathbf{d p})$ & $\mathbf{x}(\mathbf{d p})$ & & & \\
\hline Sintomas depressivos & $3,80(2,15)$ & $4,40(3,50)$ & 0,370 & 0,21 \\
Sintomas de ansiedade & $7,50(6,43)$ & $7,50(6,35)$ & 1,000 & 0,00 \\
\hline
\end{tabular}

*Diferença significativa $(\mathrm{p}<0,05)$ - Teste $t$ de student dependente. Fonte: Autores.

Nota-se que o desempenho em equilíbrio $(\mathrm{p}=0,01)$ apresentou alteração entre o primeiro $(\mathrm{x}=10,50)$ e último $(\mathrm{x}=11,10)$ encontro de intervenção, manifestando significância estatisticamente significativa $(p=0,005)$. Observa-se que a magnitude da diferença foi média $(\mathrm{d}=0,69)$.

Tabela 4: Efetividade da DT em equilíbrio.

\begin{tabular}{|c|c|c|c|c|}
\hline \multirow{2}{*}{ Variáveis } & Pré-teste & Pós-teste & \multirow{2}{*}{ p-valor } & \multirow{2}{*}{ d } \\
\hline & $x(d p)$ & $x(d p)$ & & \\
\hline Equilíbrio & $10,50(0,85)$ & $11,10(0,88)$ & $0,005 *$ & 0,69 \\
\hline
\end{tabular}

*Diferença significativa $(\mathrm{p}<0,05)$ - Teste t de student dependente. Fonte: Autores.

\section{Discussão}

Este estudo teve como objetivo avaliar a eficácia de um protocolo de DT associado a estratégias cognitivas de associação e imagem mental no equilíbrio, cognição, ansiedade e nos sintomas depressivos de idosos. Os principais achados revelaram que 
o programa de intervenção em DT provocou efeito positivo somente sobre desempenho em equilíbrio. Já para as habilidades cognitivas, sintomas depressivos e sintomas de ansiedade, a intervenção em DT não teve efeito significativo.

Ao analisar os dados encontrados na presente pesquisa constatou-se que houve uma melhora significativa no equilíbrio dos idosos participantes após o programa em DT. A combinação de atividades que envolvam o equilíbrio e a cognição devem ser desenvolvidas com idosos, visto que o elevado nível de quedas destes indivíduos está diretamente relacionado as alterações de equilíbrio do envelhecimento (Milisen, Detroch, Bellens, Braes, Dierickx, Smeulders, Teughels, et al, 2004). Além disso, intervenções em DT para melhora do equilíbrio já haviam sido sugeridas como intervenção eficaz tanto em idosos saudáveis (Granacher, Muehlbauer, Bridenbaugh, Wolf, Roth, Gschwind, Wolf, Mata \& Kressig, 2012; Pichierri, Murrer \& Bruim, 2012) quanto em idosos com Doença de Parkinson e Alzheimer (Christofoletti, 2010; Pedroso, Coelho, Santos-Galduróz, Costa, Gobbi \& Stella, 2012). Inclusive, uma revisão de literatura realizada por Siqueira, Antunes, Nascimento Júnior e Oliveira (2019), apontou que o treinamento de equilíbrio com tarefa cognitiva simultânea é melhor que o treinamento de equilíbrio convencional para o ganho de equilíbrio sobre condição de DT em idosos.

Nesta pesquisa não foi possível observar efeito da intervenção sobre a cognição. Apesar de Castaño e Albany (2019) ressaltarem que o treinamento de força em DT poderia catalisar esse processo e gerar adaptações cognitivas positivas, outros estudos (Cassilhas, Viana, Grassmann, Santos, Santos, Tufik \& Mello, 2007; Liu-Ambrose, Nagamatuso, Voss, Khan \& Handy, 2012) destacam que as adaptações cognitivas começam a ser tangíveis após quatro a seis meses de intervenção. Vale lembrar que em nosso estudo, foi realizado apenas quatro semanas de intervenção, uma sessão por semana, o que pode ter sido insuficiente para eficácia nos resultados.

Corroborando com a presente pesquisa, o estudo de Sartori et al. (2017), ao avaliarem 33 idosos divididos em dois grupos, sendo um grupo controle $(n=19)$ e um grupo intervenção $(n=14)$ com média de idade de 78 anos e 77 anos respectivamente, também não observaram diferença estatisticamente significativa nos pontos da GDS após a aplicação de um protocolo DT. Em nosso estudo, não encontramos melhoras no escore da GDS após a intervenção com DT.

Não há na literatura protocolos que indiquem de maneira obrigatória as tarefas e o período que deve se realizar o treinamento em DT, mas é importante que o profissional da saúde se guie pelas evidências cientificas e individualidade de cada idoso, considerando o contexto social em que vive e as tarefas do dia-a-dia, a fim de alcançar melhoras na sua capacidade funcional e participação (Mendel, Barbosa \& Sasaki, 2015).

Mesmo diante dos importantes resultados encontrados nessa pesquisa, ela possui algumas limitações: primeiro, o pequeno tamanho amostral, o que impede uma possível maior generalização dos dados; segundo, a falta de um grupo controle, para comparações com o grupo experimental.

\section{Conclusão}

A partir dos resultados obtidos, pode-se concluir que o programa de intervenção em DT em quatro semanas provocou efeito positivo somente sobre o desempenho em equilíbrio dos idosos. No entanto, não se mostrou eficaz para as habilidades cognitivas, sintomas depressivos e sintomas de ansiedade.

Do ponto de vista prático, destaca-se a importância da DT para o desenvolvimento e manutenção do equilíbrio do idoso, que é uma capacidade física fundamental para a independência funcional e realização das atividades da vida diária. Com isso, profissionais de educação física e fisioterapia devem prescrever tais atividades com idosos diariamente.

\section{Referências}

Bowen, A., Wenman, R., Mickelborough, J., Foster, J., Hill, E., \& Tallis, R. (2001). Dual-task effects of talking while walking on velocity and balance following a stroke. Age Ageing, 30(4):319-23. 
Brucki, S. M. D., Nitrini, R., Caramelli, P., Bortolucci, P. H. F., \& Okamoto, I. H. (2003). Sugestões para o uso do mini-exame do estado mental no Brasil. Arquivos de Neuropsiquiatria, 61(3): 777-81.

Casey, D. A. (2017). Depression in Older Adults: A Treatable Medical Condition. Primary Care, 44(3):499-510.

Cassilhas, R. C., Viana, V. A. R., Grassmann, V., Santos, R. T., Santos, R. F., Tufik, S., \& Mello, M. T. (2007). The impact of resistance exercise on the cognitive function of the elderly. Medice and Science in Sports Exercise, 39(8):1401-7.

Castaño, A., \& Albany, L. (2019). Influência do treinamento de força, com e sem dupla tarefa, em idosos saudáveis: aspectos físicos, morfofuncionais e cognitivos [Dissertação de Mestrado]. Universidade Estadual de Campinas.

Christofoletti, G. (2010). Influência da dupla-tarefa no equilíbrio de pacientes com doença de Parkinson e demência do tipo Alzheimer [Tese de Doutorado].: Universidade Estadual de Campinas.

Crook, T. H., Feher, E. P., \& Larrabee, G. J. (1992). Assessment of Memory Complaint in Age-Associated Memory Impairment: The MAC-Q. International Psychogeriatrics, 4(2):165-76.

Fatori, C. O., Leite, C. F., Souza, L. A. P. S., \& Patrizzi, L. J. (2015). Dupla tarefa e mobilidade de idosos ativos. Revista Brasileira de Geriatria e Gerontologia, 18(1):29-37.

Fechine, B. R. A., \& Trompieri, N. (2012). O processo de envelhecimento: as principais alterações que acontecem com o idoso com o passar dos anos. Interscience Place, 1(20):106-32.

Folstein, M. F., Folstein, S. E., \& McHugh, P. R. (1975). Mini mental State: a practical method for grading the cognitive state of patients for clinician. Journal of Psychiatric Research, 12(1), 189-98.

Ghai, S., Ghai, I., \& Effenberg, A. O. (2017). Effects of dual tasks and dual-task training on postural stability: a systematic review and meta-analysis. Clinical Interventions in Aging, 12:557-77.

Granacher, U., Muehlbauer, T., Bridenbaugh, S. A., Wolf, M., Roth, R., Gschwind, Y., Wolf, I., \& et al. (2012). Effects of a Salsa Dance Training on Balance and Strength Performance in Older Adults. Gerontology, 58(4):305-12.

Kim, W., Kim, B., Chang, S., Lee, D., \& Bae, J. (2020). Relationship between subjective memory complaint and executive function in a community sample of South Korean elderly. Psychogeriatrics, 20(6):850-7.

Klojčnik, M., Kavcic, V., \& Vukman, K. B. (2017). Relationship of Depression With Executive Functions and Visuospatial Memory in Elderly. International Journal of Aging \& Human Development, 85(4):490-503.

Liu-Ambrose, T., Nagamatuso, L. S., Voss, M. W., Khan, K. M., \& Handy, T. C. (2012). Resistance training and functional plasticity of the aging brain: a 12month randomized controlled trial. Neurobiology of Aging, 33(8):1690-8.

Macena, W. G., Hermano, L. O., \& Costa, T. C. (2018). Alterações fisiológicas decorrentes do envelhecimento. Revista Mosaicum, 15(27):223-38.

Martiny C., Silva, A. C. O., Nardi, A. E., \& Pachana, N. A. (2011). Tradução e adaptação transcultural da versão brasileira do Inventário de Ansiedade Geriátrica (GAI). Archives of Clinical Psychiatry, 38(1):8-12.

Mak, W. W. S., Chan, R. C. H., \& Yau, S. S. W. (2019). Brief Psychoeducation Program to Enhance Recovery Knowledge and Attitudes of Mental Health Service Providers and Users: Randomized Controlled Trials. Administration and Policy in Mental Health, 46(2):200-8.

Mendel, T; Barbosa, W. O; \& Sasaki, A. C. (2015). Dupla Tarefa como estratégia terapêutica em fisioterapia neurofuncional: uma revisão de literatura. Acta Fisiátrica, 22(4):206-11.

Milisen, K., Detroch E., Bellens, K., Braes, T., Dierickx, K., Smeulders, W., Teughels, S. \& et al. (2004). Falls among community-dwelling elderly: a pilot study of prevalence, circumstances and consequences in Flanders. Tijdschr voor Gerontologie en Geriatrie, 35(1):15-20.

Miranda, D. C; Brucki, S. M. D; \& Yassuda, M. S. (2018). The Mini-Addenbrooke's Cognitive Examination (M-ACE) as a brief cognitive screening instrument in Mild Cognitive Impairment and mild Alzheimer's disease. Dementia \& Neuropsychologia, 12(4):368-73

Nakano, M.M. (2007). Versão brasileira da Short Physical performance battery SPPB: Adaptação cultural e estudo da confiabilidade [Dissertação de Mestrado] São Paulo: Universidade Estadual de Campinas.

Norouzi, E., Vaezmosavi, M., Gerber, M., Pühse, U., \& Brand, S. (2019). Dual-task training on cognition and resistance training improved both balance and working memory in older people. The Physician and Sportsmedicine, 47(4):471-8.

Oliva, R. N. P., Machado, F. S. M., Rodrigues, V. D., Leão, L. L., \& Monteiro-Júnior, R. S. (2020). The effect of dual-task training on cognition of people with different clinical conditions: An overview of systematic reviews. IBRO Reports, 9:24-31.

Pachana, N. A., Byrne, G. J., Siddle, H., Koloski, N., Harley, E., \& Arnold, E. (2007). Development and validation of the Geriatric Anxiety Inventory. International Psychogeriatrics, 19(1):103-14.

Paradela, E. M. P., Lourenço, R. A., \& Veras, R. P. (2005). Validação da escala de depressão geriátrica em um ambulatório geral. Revista de saúde pública, 39:918-23.

Pedroso, R. V., Coelho, F. G. M., Santos-Galduróz, R. F., Costa, J. L. R., Gobbi, S., \& Stella, F. (2012). Balance, executive functions and falls in elderly with Alzheimer's disease (AND): a longitudinal study. Archives of Gerontology and Geriatrics, 54(2):348-51. 
Research, Society and Development, v. 10, n. 10, e449101018675, 2021

(CC BY 4.0) | ISSN 2525-3409 | DOI: http://dx.doi.org/10.33448/rsd-v10i10.18675

Pichierri, G.; Murer, K.; Bruin E. D. (2012). A cognitive-motor intervention using a dance video game to enhance foot placement accuracy and gait under dual task conditions in older adults: a randomized controlled trial. BMC Geriatrics, 12:74.

Sartori, R., Souza, C. M., Dias, F. M., Vidal, A. P. C., \& Dias, F. M. V. (2017). Efeitos da Intervenção motora com tarefa dupla na cognição e presença de depressão em idosos residentes em instituição de longa permanência. Fisioterapia Brasil, 18(4):391-400.

Siqueira, J. F., Antunes, M. D., Nascimento Júnior, J. R. A., \& Oliveira, D. V. (2019). Efeitos da prática de exercício de dupla tarefa em idosos com doença de alzheimer: revisão sistemática. Saúde e Pesquisa, 12(1):197-202.

Varela-Vásquez, L. A., Minobes-Molina, E., \& Jerez-Roig, J. (2020). Dual-task exercises in older adults: A structured review of current literature. Journal of Frailty, Sarcopenia and Falls, 5(2):31-7.

Wiel, A. B., Gussekloo, J., Craen, A. J. M., Exel, E. V., Bloem, B. R., \& Westendorp, R. G. J. (2003). Walking and talking as predictors of falls in the general population: the Leiden 85-Plus Study. Journal of American Geriatrics Society, 51(10):1466-71.

Yesavage, J. A., Brink, T. L., Rose, T. L., Lum, O., Huang, V., Adey, M., \& Leirer, V. O. (1982). Development and validation of a geriatric depression screening scale: a preliminary report. Journal of psychiatric research, 17(1):37-49. 